\title{
Acute Perforated Appendicitis
}

National Cancer Institute

\section{Source}

National Cancer Institute. Acute Perforated Appendicitis. NCI Thesaurus. Code C78178.

Acute appendicitis with gangrenous changes resulting in the rupture of the appendiceal

wall. The appendiceal wall rupture causes the release of inflammatory and bacterial

contents from the appendiceal lumen into the abdominal cavity. 